\title{
RNA viruses: hijacking the dynamic nucleolus
}

\section{Julian A. Hiscox}

Abstract | The nucleolus is a dynamic subnuclear structure with roles in ribosome subunit biogenesis, mediation of cell-stress responses and regulation of cell growth. The proteome and structure of the nucleolus are constantly changing in response to metabolic conditions. RNA viruses interact with the nucleolus to usurp host-cell functions and recruit nucleolar proteins to facilitate virus replication. Investigating the interactions between RNA viruses and the nucleolus will facilitate the design of novel anti-viral therapies, such as recombinant vaccines and therapeutic molecular interventions, and also contribute to a more detailed understanding of the cell biology of the nucleolus.

Positive-strand RNA virus A virus with a single-stranded RNA genome that can function as an mRNA without further transcription.

Negative-strand RNA virus A virus with a single-stranded RNA genome that is

'antimessenger' sense and therefore does not encode mRNA

Fibrillar centre

(FC). A compartment of the nucleolus found within most metazoan nucleoli, but not usually found in lower

eukaryotes, which is organized around multiple rRNA genes in tandem arrays found at several chromosomal loci.
Institute of Molecular and Cellular Biology, Faculty of Biological Sciences, and Astbury Centre for Structural Molecular Biology, Garstang Building, University of Leeds, Leeds, LS2 9JT, UK. e-mail:j.a.hiscox@leeds.ac.uk doi:10.1038/nrmicro1597
Viruses are obligate intracellular parasites and this characteristic is exemplified by RNA viruses ${ }^{1}$. The genomes of RNA viruses are usually smaller than the genomes of DNA viruses, and RNA viruses are therefore more reliant on subverting host-cell proteins, and cellular structures and functions to facilitate virus replication. One structure that is targeted by RNA viruses is the nucleolus, a dynamic subnuclear structure. Most DNA viruses and retroviruses replicate in the nucleus, and therefore it makes sense that these viruses target nuclear structures. But as the primary site of replication of positive-strand RNA viruses and most negative-strand RNA viruses is the cytoplasm, the rationale for RNA viruses targeting nuclear structures is not immediately obvious. However, as discussed in this Review, recent research that capitalizes on advances in proteomics and cellular imaging techniques is beginning to increase our understanding of the mechanisms that RNA viruses use to subvert host-cell nucleoli.

\section{Nucleolar structure and function}

The nucleolus was one of the first subcellular structures to be identified by early users of the light microscope, appearing as a highly refractive black $\operatorname{dot}(\mathrm{s})$ in the nucleus of the cell ${ }^{2}$, which reflects its dense protein content (FIG. 1). More detailed analysis of mammalian cells by electron microscopy indicated that the nucleolus could be divided into three discrete regions that reflect its primary role in ribosome subunit biogenesis ${ }^{3}$. These regions can be viewed as compartments formed by the functional association of macromolecules and comprise fibrillar centres, surrounded by dense fibrillar components, which in turn are surrounded by a granular component (FIG. 1). The composition and structure of the nucleolus varies between different biological kingdoms. In mammalian cells the nucleolus is present during interphase but disintegrates and reforms in mitosis ${ }^{4}$. In yeast the nucleolus is present throughout the cell cycle and is proximal to the nuclear envelope. Plant-cell nucleoli have a fourth nucleolar structure, which has been referred to as a nucleolar vacuole or cavity ${ }^{5}$.

For many years the classic view of the nucleolus focused on its crucial function in ribosomal subunit biogenesis. Ribosomes are composed of a large and small subunit, which each contain their own ribosomal RNA (rRNA) molecules. In eukaryotes there are four different rRNA molecules (28S, 5S and 5.8S rRNAs in the large subunit and 18S rRNA in the small subunit) and $>80$ proteins. Transcription of the primary $45 \mathrm{~S}$ pre-rRNA molecule from rDNA initiates in the fibrillar centre, and the nascent molecules are distributed in a 'Christmas tree'-like arrangement. Processing of the 45S pre-rRNA (into 5.8S, $18 \mathrm{~S}$ and $28 \mathrm{~S}$ rRNAs) occurs at, or near, the border between the dense fibrillar component and the fibrillar centre, and RNA assembly into ribosomal subunits occurs in the granular component before export into the cytoplasm. An additional feature is the perinucleolar compartment, which has also been implicated in RNA metabolism ${ }^{6}$ and is associated with chromatin. Although studies of the nucleolus have focused on ribosomal precursor RNA, many other RNA species have been identified in the nucleolus including several precursor tRNA species and the signal-recognition particle ${ }^{7}$. 
Dense fibrillar component (DFC). A compartment of the nucleolus that contains newly synthesized pre-ribosomal RNA and a collection of proteins. DFCs are normally associated with FCs.
The nucleolus has also been proposed as a sensor of cellular stress, and one of the main molecules involved in this process is the tumour suppressor protein $\mathrm{p} 53$, which accumulates in the nucleus when cells are subjected to stressful stimuli, such as ultraviolet (UV) light and inhibitor molecules. For example, the inhibition of proteasome activity results in the accumulation of p53 in the nucleolus and its association with nucleo$\operatorname{lin}^{8}$, and similar results have been found when cells are exposed to ionizing radiation ${ }^{9}$. Definitive experiments later showed that nucleolar disruption is a common factor in all stress responses that involve p53, and that impairment of nucleolar function leads to $\mathrm{p} 53$ stabilization $^{10,11}$.
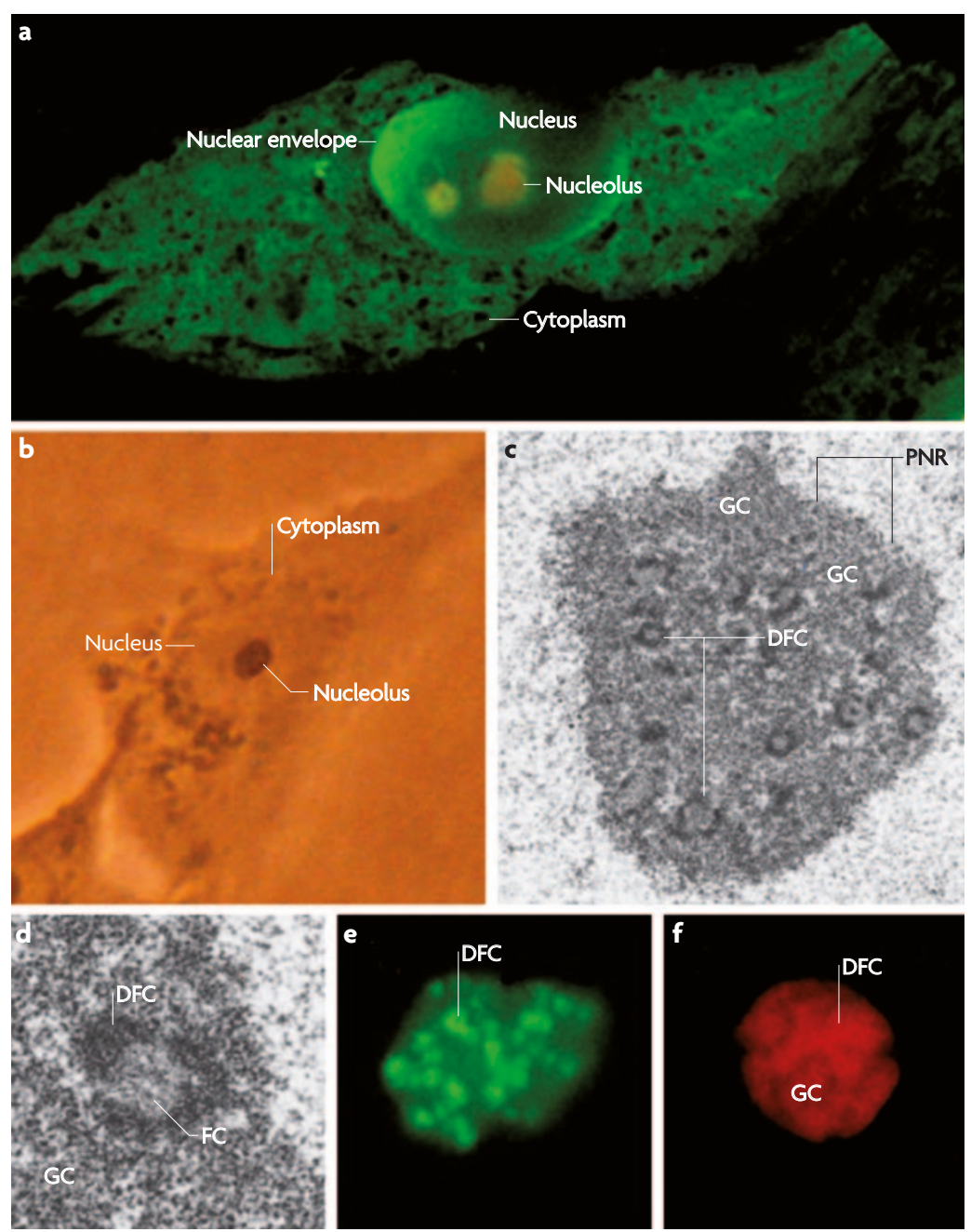

Figure 1 | The structure of the nucleolus. a | Confocal microscopy image of a mammalian cell showing the relative positions of the cytoplasm, nucleus and nucleolus. The nucleus and the nucleolus are separated from the cytoplasm by the nuclear envelope. $\mathbf{b}$ |Bright-field microscopy image of a live mammalian cell showing the nucleolus as a highly refractive structure in the nucleus. c-d | Electron microscopy images of the mammalian nucleolus highlighting distinct structures, including the fibrillar centre (FC), dense fibrillar component (DFC), granular component (GC) and perinucleolar region (PNR). Many diagrams of the nucleolus show only a single FC and DFC when, in fact, there are many FCs and DFCs in one nucleolus. $\mathbf{e}-\mathbf{f} \mid$ Confocal images of the nucleolus showing how fluorescent fusion proteins of nucleolar proteins can be used as markers for the different regions of the nucleolus including the DFC (EGFP-fibrillarin) (e) and the GC and DFC (DS-Red-B23) (f).

\section{The dynamic nucleolus}

Dramatic inroads have been made into the elucidation of nucleolar function through extensive proteomic analysis of human nucleoli (which indicate the presence of $>700$ nucleolar proteins $)^{12}$ and plant nucleoli ${ }^{13}$, in some cases under differing metabolic conditions ${ }^{14}$. These studies have contributed to the emerging concept of the nucleolus as a dynamic compartment that is involved in the control of numerous cellular functions. Time-lapse video microscopy and fluorescence recovery after photobleaching (FRAP) and fluorescence loss in photo-bleaching (FLIP) assays (BOX 1) have shown that the content of the nucleolus is constantly changing ${ }^{3}$.

Although most of the mammalian nucleolar proteome is associated with ribosome manufacture and rRNA processing (76 ribosomal proteins are located in the nucleolus), many other groups of nucleolar proteins have discrete functions. For example, 25 nucleolar proteins are specifically related to cell-cycle control. Although the number of nucleolar proteins identified has increased with the use of more sophisticated analytical techniques, the relative proportion of proteins in each functional class has remained the same ${ }^{14,15}$. The interaction between different nucleolar proteins is central to coordinating the functions of this compartment. The multifunctional nature of nucleolar proteins and their numerous protein interactions illustrates an overriding principal of nucleolar architecture: that it is an amalgamation of many protein-protein and protein-nucleic-acid interactions, and disruption of these interactions can cause aberrant nucleolar function and possibly lead to disease ${ }^{16}$.

Three distinct subsets of nucleolar proteins have been identified that reflect the dynamic nature of the nucleolar proteome ${ }^{12,14}$. First, proteins that are mainly located in the nucleolus, such as the major nucleolar proteins fibrillarin, which is a component of a nucleolar small nuclear ribonucleoprotein that is involved in rRNA processing; B23.1, which is a putative ribosome assembly factor; and nucleolin, which is involved in the processing of precursor rRNA. Second, proteins that traffic from the nucleolus to the cytoplasm and so are not always resident in the nucleolus, such as ribosomal proteins. Third, proteins for which residence in the nucleolus is time- and/or condition-dependent, such as the 25 nucleolar proteins with functions that are specifically related to cell-cycle control, including cyclin-dependent kinase (CDK)2, CDK7, CDK9, cell division cycle-2-like protein kinase-5 (CDC2L5), CIP1/p21 and Aurora B ${ }^{14}$. However, other nucleolar proteins can have cell-cycle-related functions, including nucleolin and B23.1 (REF. 17).

\section{Targeting proteins to and from the nucleolus}

The rules and signals that govern the nuclear localization and nuclear export of proteins are well defined. Nuclear-localization signals (NLSs) can be classified into several categories. Most motifs identified so far, including the pat 4 and pat7 motifs and bipartite NLSs, are composed of basic amino acids of a given sequence 
Granular component (GC). A compartment in the nucleolus that contains nearly complete pre-ribosomal particles destined for the cytoplasm, and which forms the main visible feature under the light microscope. Several FCs and DFCs are located in this structure and their frequency can depend on the metabolic state of the cell.

\section{Interphase}

The period between two

mitotic divisions.

Perinucleolar compartment A compartment that is usually found in transformed cells and is composed of fibres with a diameter of $80-180 \mathrm{~nm}$ that are in direct contact with the nucleolus. This structure might have a role in RNA processing.

Fluorescence recovery after photo-bleaching

(FRAP). A technique used to measure the movement of

fluorescent-tagged molecules over time in vivo. Specific

regions in a cell are irreversibly photobleached using a laser

fluorescence is restored by diffusion or trafficking of

fluorescent-tagged unbleached molecules into the bleached area

Fluorescence loss in photobleaching

(FLIP). The continuous photobleaching of a defined area. Changes in fluorescence in the non-bleached region represent the sum of all movements of the fluorescent molecules, whether by diffusion or trafficking. FLIP is different to FRAP in that, in the latter, only one (brief) photo-bleach occurs. However, both techniques are used to measure the kinetics of protein movement.

\section{Box 1 Studying the nucleolus by direct imaging}

Several techniques have been used to study the nucleolus, from proteomics to imaging. The three most common techniques for visualizing the trafficking of nucleolar proteins are fluorescence recovery after photo-bleaching (FRAP), fluorescence loss in photo-bleaching (FLIP) and confocal microscopy of fixed cells. When used with cellular or viral nucleolar proteins that have been tagged with a fluorescent fusion protein (such as enhanced green fluorescent protein; EGFP) FRAP and FLIP allow measurements of dynamic trafficking. In FRAP analysis, a portion of the nucleolus (or the whole nucleolus) is irreversibly photo-bleached by a single, high-powered spot laser pulse. The recovery of the fluorescent signal in the bleached area is the consequence of movement of the EGFP-fusion protein back into the bleached area. This is recorded by sequential imaging scans, and the mobility of the protein can be calculated from the kinetics of fluorescence recovery (see the figure). In FLIP analysis, a portion of, or a whole nucleolus, can be continuously photo-bleached in a manner similar to FRAP. In this way, the trafficking of several nucleolar proteins, including nucleolin, fibrillarin and B23.1, has been examined ${ }^{3,112-115}$.

The figure shows cells expressing GFP-fibrillarin before bleaching and during recovery after bleaching of the entire nucleolus. The images were taken at the indicated times after the end of the bleach pulse. The area of the bleach spot is indicated with a circle. The indicated area is shown enlarged in pseudocolour in the lower panels. Scale bar $=5 \mu \mathrm{m}$. The figure is reproduced with permission from REF. 113 @ (2000) Macmillan Magazines Ltd.
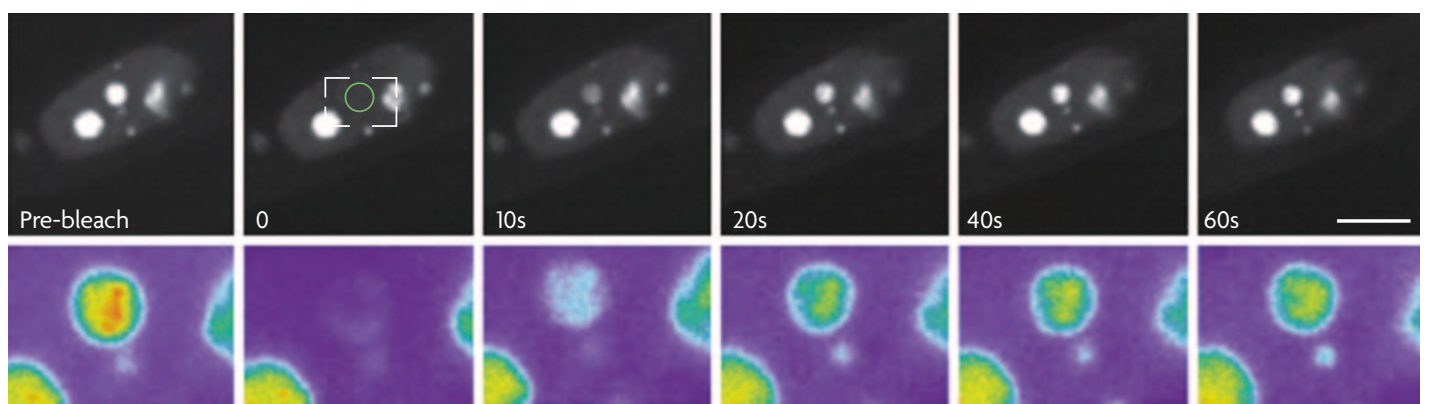

length ${ }^{18-20}$. The pat 4 motif consists of a continuous stretch of (usually) four basic amino acids, and the pat7 motif starts with a proline residue and is followed by six amino acids, of which three of the last four are basic. As its name suggests, a bipartite signal contains two separate motifs, both of which are rich in basic amino acids. Protein nuclear-export signals (NESs) also vary, but one of the most common and well characterized NESs is an $\sim 11$ amino-acid, leucine-rich signal, typified by LX(2,3)LX(2,3)LXL, where several hydrophobic amino acids can substitute for leucine. The number of spacer residues (X) is indicated in brackets $^{19,21}$.

By contrast, the signals that govern nucleolar localization and retention are not well defined ${ }^{22}$. The motifs involved are usually rich in arginine and lysine residues, however, there is no immediately obvious consensus sequence or structure. Presumably, proteins that localize to the nucleolus can also have nuclear-import and, if necessary, nuclear-export motifs. The nucleolar-localization signals (NoLSs) that have been identified so far can be grouped into those that contain single motifs and those that contain multiple motifs ${ }^{23}$. For example, single motifs include MQRKPTIRRKNLRLRRK identified in survivindeltaEx3, a splice variant of the anti-apoptotic protein survivin ${ }^{24}$, and the RSRKYTSWYVALKR motif of fibroblast growth factor-2 (REF. 25). Alternatively, signals can take the form of an interaction between several functional domains of a protein, for example, in two components of the $60 \mathrm{~S}$ ribosomal subunit - the human ribosomal L7a protein ${ }^{26}$ and ribosomal protein L5 (REF. 27). Nucleolar localization can also be regulated by binding small effector molecules, such as nucleostemin binding to $\mathrm{GTP}^{28}$. Whether proteins localize, target, accumulate or are retained in the nucleolus is unknown, and the targeting signals that are associated with nucleolar proteins have been called not only NoLSs but also nucleolar-targeting signals and nucleolar-retention signals.

In many cases, proteins that localize to the cytoplasm and the nucleus or nucleolus contain multiple signals that determine their subcellular localization ${ }^{29-32}$. This highlights the difficulty in identifying NoLSs, as many proteins that localize to the nucleolus also localize to the nucleus and contain both classic NLSs and NoLSs, the sequences of which can overlap. Nucleolar localization of a protein can be governed by several factors, including whether the protein associates with another nucleolar-bound protein or alternatively traffics to the nucleolus on its own or associates with RNA transcripts that are being transcribed in the nucleolus. Clearly, combinations of these possibilities could and do occur, compounding the difficulty in predicting and defining NoLSs.

\section{Viruses and the nucleolus}

The interaction between viruses and the nucleolus is a pan-virus phenomenon, which is exhibited by DNA viruses, retroviruses and RNA viruses. Whereas DNA viruses and retroviruses that interact with the nucleolus replicate in the nucleus where the nucleolus is immediately accessible, why RNA viruses, particularly positivestrand RNA viruses, interact with the nucleolus when 


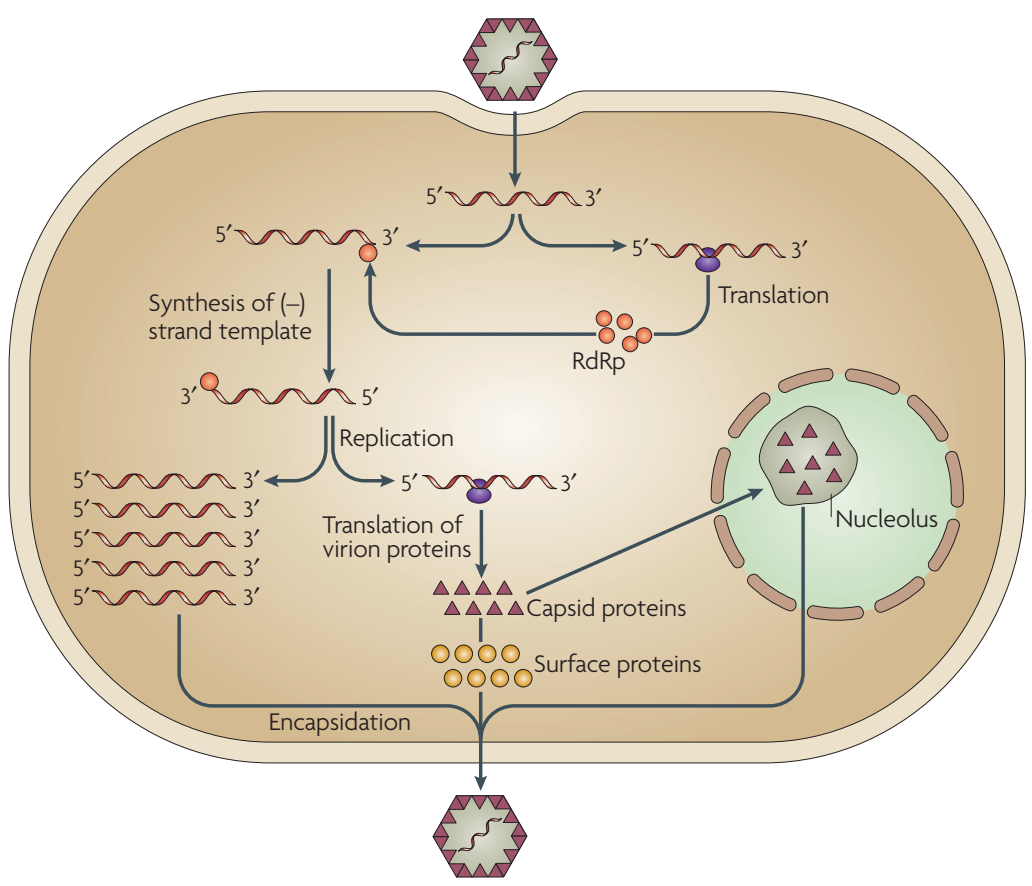

Figure 2 | RNA viruses and the nucleolus. Schematic representation of a generic replication strategy used by a positive-strand RNA virus showing localization of the capsid protein to the nucleolus. The virus enters the cell and the genome is uncoated and then translated by ribosomes to produce the viral RNA-dependent RNA polymerase (RdRp). The viral genome is therefore messenger sense and also functions as a template for the synthesis of negative-strand RNA. The RdRp uses the negative strand (or anti-genome) as a catalytic template for the production of new genomic RNA. Therefore, there is usually a molar excess (between 10:1 and 100:1) of positivestrand RNA over negative-strand RNA in infected cells. Positive-strand RNA viruses can either be enveloped or non-enveloped. However, they all produce capsid proteins for binding to viral RNA. These proteins have different names, including capsid,

nucleocapsid and nucleoproteins, but all have similar functions. Negative-strand RNA viruses have a more complex life cycle in that the viral genome is not messenger sense and therefore the virus contains the enzymes needed to initiate the transcription of mRNAs and to facilitate replication ${ }^{103}$.

Nuclear-pore complex A large multiprotein complex that forms a channel in the nuclear envelope of a eukaryotic cell joining the inner and outer nuclear membranes and allowing transport of proteins to and from the nucleus. the site of viral-genome replication is in the cytoplasm (FIG. 2) is less intuitive. For positive-strand RNA viruses, viral proteins must transit through the nuclear-pore complex (BOX 2) to enter and exit the nucleus. Passage in and out of the nucleolus and nucleus is crucial for virus biology because if the viral proteins that are required for cytoplasmic functions, such as RNA synthesis and encapsidation, are sequestered in the nucleolus or nucleus, then progeny virus production will be affected.

Although studies of the replication of positivestrand RNA viruses in enucleated cells indicated that there is no direct nuclear component in the life cycle of these viruses ${ }^{33,34}$, the enucleation in these experiments was not $100 \%$ and, although the viruses did replicate, replication was decreased. Conflicting results were also obtained for viruses that belong to the same viral group. For example, the murine coronavirus mouse hepatitis virus was reported to replicate in enucleated cells ${ }^{33}$ whereas the avian coronavirus infectious bronchitis virus (IBV) requires the nucleus and host-cell factors to replicate ${ }^{34}$.
New data are now emerging that support the view that many classic positive-strand RNA viruses interact with the nucleus and nucleolus to facilitate virus replication. One of the most well-studied viruses in terms of viral interactions with the nucleolus is HIV. Although HIV has clearly defined cytoplasmic and nuclear replication strategies, the virus has a positive-sense RNA genome - the viral capsid contains two copies of positive-sense RNA, but these are reverse transcribed in the cytoplasm and then trafficked to the nucleus, where the new genome is transcribed and trafficked back to the cytoplasm.

Although many RNA-virus proteins have been shown to localize to the nucleolus, most attention has focused on capsid and RNA-binding proteins, which have been shown to localize to the nucleolus in infected cells or when overexpressed. These include proteins from positive-strand RNA viruses, including the coronavirus nucleocapsid protein ${ }^{35-39}$, the arterivirus nucleocapsid protein ${ }^{40,41}$, the alphavirus capsid protein $^{42}$ and non-structural protein nsP2 (REF. 43), the dengue virus core protein $^{44}$, umbravirus ORF3 (REF. 45), the potato leafroll virus capsid protein ${ }^{46}$ and proteins from negative-strand RNA viruses, including the Newcastle disease virus (NDV) matrix protein ${ }^{47}$ and the influenza virus nucleoprotein ${ }^{48,49}$. For many years it was believed that these findings were phenomenological, and that viral capsid and RNA-binding proteins might simply localize to the nucleolus because they diffuse through the nuclear-pore complex and associate with compartments in the nucleus that have high RNA contents, and therefore with the nucleolus because it is transcriptionally active. In this case, localization to the nucleolus would have no physiological consequences for the virus or the cell. However, RNAvirus replication is error prone and selection pressure might apply to such a fortuitous localization (given the $\sim 700$ nucleolar proteins and their diverse roles), with the concomitant effect that the virus could select for changes in its genome that ultimately disrupt nucleolar function or recruit nucleolar proteins to aid virus replication.

There is a potential correlation between the nucleolar localization of a viral protein and the loss of an essential nucleolar function ${ }^{16,50}$. The molecular mechanisms that are responsible for this effect are unknown, but the displacement and relocalization of nucleolar proteins by viral proteins could increase or decrease the nucleolar, nuclear or cytoplasmic pool of these proteins. Certainly, the accumulation of viral proteins in the nucleolus could potentially cause volume exclusion or crowding effects, which have been proposed to have a fundamental role in the formation of nuclear compartments including the nucleolus ${ }^{51}$. Therefore, disruption of nucleolar architecture and function might be common in virus-infected cells if viral proteins target the nucleolus or a stage of the virus lifecycle disrupts nucleolar proteins. For example, poliovirus infection results in the selective redistribution of nucleolin from the nucleolus to the cytoplasm ${ }^{52}$ and inactivation of the nucleolar protein UBF (upstream binding factor), which shuts off RNA polymerase I transcription in the host cell ${ }^{53}$. The infection of cells with IBV has 
been shown to disrupt nucleolar architecture ${ }^{37}$ and cause arrest of the cell cycle in G2 and failure of cytokinesis ${ }^{36,54,55}$. The IBV and arterivirus nucleocapsid proteins associate with nucleolin ${ }^{55}$ and fibrillarin ${ }^{56}$, respectively. Similarly, the HIV-1 Rev protein has been shown to localize to the DFCs and GCs, and overexpression of Rev alters the nucleolar architecture and is associated with the accumulation of B23.1 (REF. 57). Rev overexpression also correlates with an increase in the proportion of cells in the G2 and $M$ phases of the cell cycle and cells that have failed cytokinesis ${ }^{57,58}$. Indeed, changes in nucleolar architecture have been observed in lymphocytes from patients infected with HIV-1 and this correlated with a loss of cell-cycle control $^{59}$. From a therapeutic perspective, administering interleukin-2 can reverse the loss of cell-cycle control and might be a useful tool for HIV-1 therapy ${ }^{60}$.

Transporting RNA-virus proteins to the nucleolus Many different RNA-virus proteins localize to the nucleolus. Viral proteins that traffic to the nucleolus contain motifs that resemble host NoLSs, and therefore viral nucleolar trafficking might use a form of molecular mimicry ${ }^{61}$. The discovery of specific nucleolar-trafficking signals in viral proteins would indicate that they are functionally relevant $t^{61}$. Historically, virology has informed and driven many areas of cell biology, including the discovery of the first NLS in an SV40-virus protein ${ }^{62}$, which subsequently led to the identification and characterization of NLSs in other viral and cellular proteins ${ }^{18}$. However, similar to nucleolar-targeting signals in host cells, no overall conserved nucleolar trafficking motif has been identified in viral proteins.

In some virus proteins both NLSs and NoLSs function in concert to direct a protein to the nucleolus. The nucleocapsid protein of the arterivirus porcine reproductive and respiratory syndrome virus (PRRSV) localizes to the nucleolus ${ }^{41}$ and has been shown to contain two potential NLSs: a pat4 motif and a downstream pat7 motif ${ }^{63}$. Analysis revealed that a 31 amino-acid sequence that incorporates the pat7 motif could direct the

\section{Box 2 | The nuclear-pore complex}

To enter and exit the nucleus all molecules or cargoes must traverse a large macromolecular structure called the nuclear-pore complex (NPC), which is located in the nuclear envelope. This is one of the most complex cellular structures and is composed of $>30$ different proteins and, in mammalian cells, has a molecular weight of over $125 \mathrm{MDa}$. Small molecules - up to 50-60 kDa or less than $10 \mathrm{~nm}$ in diameter can diffuse passively through the NPC, but if proteins are larger than this size-exclusion limit and/or are required to move against a concentration gradient then transport requires energy-driven mechanisms. In this case, most proteins will contain the appropriate trafficking motifs such as nuclear-localization and nuclear-export signals. Nuclear-localization signals are recognized by proteins of the importin superfamily (importin $\alpha$ and $\beta$ ) that mediate protein transport across the nuclear envelope using RanGTP. Nuclearexport signals are recognized by exportins. nucleocapsid protein to both the nucleus and nucleolus ${ }^{63}$. The protein also contains a predicted NES, presumably to allow the protein to traffic back into the cytoplasm to contribute to viral function in that compartment ${ }^{61}$. Viral proteins might also traffic to the nucleolus through association with cellular proteins. For example, the hepatitis delta antigen has been shown to contain an NoLS that also corresponds to a site that promotes binding to nucleolin ${ }^{64}$. Mutating this region prevents nucleolin binding to the delta antigen and prevents nucleolar trafficking. By implication, this relates nucleolin binding to nucleolar trafficking.

Predicting viral and cellular NoLSs is problematic, and currently there is no bioinformatics software available to do so. As a retrospective example, the IBV nucleocapsid protein localizes to the nucleolus and associates with nucleolin ${ }^{35,37,55}$, therefore one would predict that this protein would contain an NoLS. Comparing the sequence of several different RNA virus NoLSs to the complete amino-acid sequence of the IBV nucleocapsid protein indicates that a basic amino-acid motif in the $\mathrm{C}$-terminal region of the protein could be considered a candidate NLS and/or NoLS. Indeed, this signal is conserved in all coronavirus nucleocapsid proteins $s^{35,36,65}$ and, on this basis, it was predicted that this motif would be involved in the pathogenesis of the severe acute respiratory syndrome coronavirus (SARS-CoV) ${ }^{65}$. However, deletion and substitution mutagenesis revealed that an eight amino-acid motif located in the $\mathrm{N}$-terminal region of the IBV nucleocapsid protein was necessary and sufficient for the protein to traffic to the nucleolus ${ }^{66}$, and the $\mathrm{N}$-terminal and central region of the SARS-CoV nucleocapsid protein, rather than the C-terminal region, contained motifs which could function as both NLSs and NoLSs ${ }^{38,39,67}$.

The fact that viral proteins contain NoLSs is a strong indication that viruses have evolved specific nucleolar functions. Evidence that the disruption of the nuclear or nucleolar trafficking of positive-strand RNA-virus proteins affects viral pathogenesis is now emerging. For example, the Semliki Forest virus non-structural protein nsP2 can localize to the nucleolus ${ }^{68}$, and disruption of this localization through a single amino-acid change results in a reduction in neurovirulence ${ }^{69}$. Such in vitro data have also been backed up by persuasive in vivo data. Mutation of the arterivirus nucleocapsid protein pat7 NLS motif in the context of a full-length clone revealed that this sequence might have a key role in virus pathogenesis in vivo as animals infected with mutant viruses had shorter duration of viraemia than with wild-type viruses ${ }^{70}$. Interestingly, reversions occurred in the mutated nucleocapsid gene sequence, and although the amino-acid sequence of the pat7 motif was altered, its function was not; this new signal was defined as a pat8 motif $^{70}$.

Similarly, point mutations in the Japanese encephalitis virus (JEV) core protein that abolished nuclear and nucleolar localization resulted in recombinant viruses with impaired replication in mammalian cells compared with wild-type virus ${ }^{71}$. Curiously, replication of recombinant viruses was not impaired in insect cells ${ }^{71}$. This 
could potentially be related to differences in nucleolar architecture $^{72}$ and proteomes between these cell types. The JEV core protein has been shown to interact with B23.1 and is translocated from the nucleolus to the cytoplasm ${ }^{73}$. Flaviviruses (such as JEV, dengue virus and West Nile virus) seem to have a nuclear stage to the synthesis of viral RNA, and several components of the viral replicase together with newly synthesized RNA have been found in the nucleus of infected cells ${ }^{74}$. One intriguing question is how such viral RNA traffics from the nucleus to the cytoplasm. Most cellular $\mathrm{mRNAs}$ are spliced, and the splicing process recruits proteins needed for efficient nuclear export ${ }^{75}$. Certain DNA viruses, such as herpesvirus saimiri, produce intron-less mRNA, and these viruses have evolved specific viral proteins (such as herpesvirus saimiri ORF57) ${ }^{76}$ that interact with the cellular mRNA-export machinery (for example, the mRNA-processing and -export factor ALY) to transport viral mRNA from the nucleus to the cytoplasm ${ }^{77,78}$, and a similar process might be used by RNA viruses. For example, in plants, tomato bushy stunt virus (TBSV) redistributes ALY from the nucleus to the cytoplasm, and this might be a way the virus mediates host-cell protein synthesis ${ }^{79}$. Also, in plants, RNA silencing, a host defence mechanism, targets viral RNAs for degradation in a sequence-specific manner, and plant viruses use several mechanisms to counteract this system. TBSV encodes a protein, P19, which interferes with this pathway. However, ALY might transport P19 from the cytoplasm to the nucleus or nucleolus and disrupt its silencing-suppressor activity ${ }^{80}$.

\section{Hijacking the nucleolus}

One of the first descriptions of an interaction between the nucleolus and viral genomes and/or proteins was the observation that plant viroid RNA was present in the nucleolus of infected cells ${ }^{81}$. Indeed, different plant-virus proteins involved in long-distance phloem-associated movement of virus particles, or with roles in binding to the RNA-virus genomes, localize to the nucleolus ${ }^{45,82-84}$, and these proteins can contain appropriate NLSs and NESs to direct protein trafficking ${ }^{31}$. 'Hijacking' the nucleolus is not exclusive to plant viruses and might also occur with mammalian viruses. Similar to the plant rhabdovirus maize fine streak virus (MFSV), the nucleocapsid and phosphoproteins of which localize to the nucleolus ${ }^{83}$, the animal negative-strand RNA virus Borna disease virus has been reported to use the nucleolus as a site for genome replication $^{85}$, and its RNA-binding protein has the appropriate trafficking signals for import and export to and from the nucleus ${ }^{86}$. The RNA of the hepatitis delta virus genome is transcribed in the nucleolus ${ }^{87}$; this is similar to the potato spindle tuber viroid, in which RNAs of opposite polarity are sequestered in different nuclear compartments, with the positive-sense RNA being transported to the nucleolus ${ }^{88}$.

As a result of their small genomes and limited coding capacities, the recruitment of cellular proteins with defined functions in RNA metabolism would be a logical step to facilitate RNA-virus infection. As nucleolar proteins have many crucial functions in cellular RNA biosynthesis, processing and translation it comes as no surprise that nucleolar proteins are incorporated into the replication and/or translation complexes formed by RNA viruses. Given that some nucleolar proteins have many functions, the same nucleolar protein might be used by a virus for different aspects of the replication pathway. One example of this is the interaction of nucleolin with picornaviruses. On the one hand, nucleolin has been shown to interact with the internal ribosome entry sites (IRESs) found in the $5^{\prime}$ non-coding region of the poliovirus genome, and this interaction stimulates IRES-dependent translation (this also occurs with the hepatitis C virus (HCV) IRES) ${ }^{89}$. As their name suggests, IRES elements promote non-cap-dependent translation ${ }^{90}$. On the other hand, nucleolin has also been shown to interact with the poliovirus $3^{\prime}$-non-coding region, which is involved in the regulation of negativestrand RNA synthesis and in the early events of virus infection $^{52}$.

Nucleolin can bind to the HCV NS5B protein, and it has been suggested that this interaction is involved in the function of NS5B during virus infection ${ }^{91}$. Interaction of picornaviruses with the nucleolus could also shut down host-cell translation. Studies indicate that the human rhinovirus $3 \mathrm{C}$ protease precursors, $3 \mathrm{CD}^{\prime}$ and $3 \mathrm{CD}$, localize in the nucleoli of infected cells early in infection and inhibit cellular RNA transcription through proteolytic mechanisms ${ }^{92}$. This general property is not restricted to human rhinoviruses and the inhibition of cellular translation has also been described for encephalomyocarditis virus $^{93,94}$.

RNA viruses, the nucleolus and disease. Nucleoli and other subnuclear structures are crucial for cell function, and disruption of these structures through interaction with viruses is likely to contribute to cellular disease, particularly through the disruption and/or displacement of nucleolar proteins. One possible link is between virus infection and the induction of autoimmune diseases. Several nucleolar proteins are associated with autoimmune disease ${ }^{95}$, for example, nucleolin is associated with systematic lupus erythematosus and fibrillarin with systemic sclerosis. Nucleolin can regulate cytokine mRNA decay indirectly through its RNA-binding activity, and inflammatory cytokines are involved in the pathogenesis of cell-mediated autoimmune diseases ${ }^{96}$. Disruption of the nucleolus and the relative subcellular distribution of nucleolar proteins through virus infection might have a role in the early events of an autoimmune response, possibly through mechanisms such as molecular mimicry.

\section{HIV - from RNA trafficking to therapy}

Studies of the biology of HIV replication have provided fundamental insights into why viruses target the nucleolus and, more importantly, how this information can be used to treat HIV infection. HIV replication involves both cytoplasmic and nuclear steps and results in unspliced, single-spliced and multiple-spliced RNAs and about 15 proteins. Several HIV proteins, including $\operatorname{Rev}^{97}$ and the transactivator Tat ${ }^{98}$, have been shown to localize 
to the nucleolus. The Rev protein exports partially or unspliced mRNA from the nucleus. Export is mediated in part by the exportin-1 nuclear-export pathway and interaction with the human Rev-interacting protein $(\text { hRIP })^{99}$. As for several other viral proteins that target the nucleolus, Rev was shown to contain a NoLS ${ }^{100,101}$ that is distinct from the NLS ${ }^{101}$. Mutation of this signal prevented the subcellular localization of Rev to the nucleolus and also reduced the efficiency of its RNAexport function ${ }^{101}$. The structure of the nucleolus also seemed to be a key factor in the localization of $\operatorname{Rev}^{102}$. Rev has been reported to undergo multimerization in both the cytoplasm and the nucleolus, but fluorescence resonance energy transfer (FRET) and FRAP studies have shown that Rev predominately forms multimers in the nucleolus ${ }^{103}$. Rev also binds to a cis-acting RNA element called the Rev-response element (RRE), which is found in all unspliced and incompletely spliced viral mRNAs, and this promotes the translocation of these mRNAs across the nuclear-pore complex.

From a therapeutic perspective, targeting the activity of the Rev protein in the nucleolus might have potential as an effective anti-viral strategy. For example, introducing an RRE decoy that localizes to the nucleolus resulted in a significant inhibition of the replication of HIV-1 in cell culture ${ }^{104}$. Similarly, the HIV Tat protein contains a NoLS ${ }^{105}$. Tat binds to a motif in HIV RNA known as the trans-activation response element (TAR). The Tat-TAR interaction increases the processivity of RNA polymerase II and promotes RNA elongation, thereby increasing the efficiency of HIV RNA transcription. Similar to the RRE decoy strategy, a chimeric nucleolar RNA-TAR decoy that localized to the nucleoli of human cells inhibited HIV-1 replication ${ }^{106}$. Taking the study further, the use of anti-HIV short hairpin RNA in cultured $\mathrm{T}$ cells and haematopoietic-stem-cell-derived monocytes demonstrated the potential therapeutic use of targeting the nucleolar stages of HIV-1 replication ${ }^{107}$.

Another interaction between HIV-1 and the nucleolus has also been exploited in the development of an antiretroviral therapy. Studies indicated that HIV-1 RNA could traffic through the nucleolus (possibly as part of an export strategy). Therefore, Michienzi and colleagues ${ }^{108}$ made use of this information and incorporated a hammerhead ribozyme into the U16 small nucleolar RNA (snoRNA) that was designed to cleave HIV-1 RNA. This construct was introduced into $\mathrm{HeLa} \mathrm{CD} 4^{+} \mathrm{T}$ cells and, when these cells were infected with HIV-1, replication of the virus was suppressed ${ }^{108}$. For therapy, this construct could be introduced into haematopoietic stems cells that have been removed from HIV-1-infected individuals, along with constructs carrying the TAR decoy and small interfering RNAs (siRNAs) to knockdown CCR5 (the co-receptor for HIV-1) ${ }^{109}$ (FIG. 3). Targeting CCR5 is a useful strategy ${ }^{110}$ because siRNA-resistant viruses will (and do) occur; however, it should not be considered a stand-alone strategy as knockdown of the receptor is not $100 \%$. Used together with conventional anti-viral chemotherapy this approach might allow 'drug holidays', or ultimately could be used in combination with anti-viral drugs or when drug-resistant viruses are present.
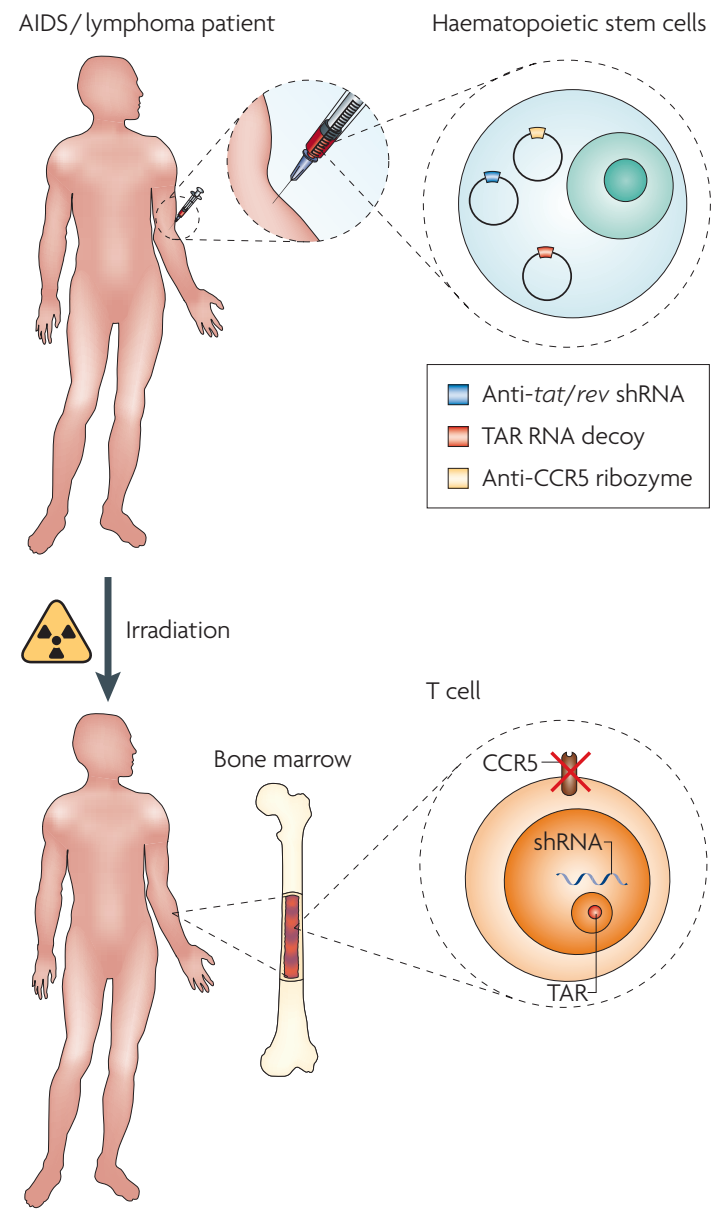

Figure $3 \mid \mathrm{HIV}$ and the nucleolus - a potential therapeutic strategy? Our knowledge of the interaction between HIV and the nucleolus is leading to the design of novel therapeutic strategies, and has been pioneered by John Rossi's group. Although these approaches are currently targeted at patients with both AIDS and lymphoma they might be applicable to all individuals infected with HIV-1. Haematopoietic stem cells are taken from infected individuals and treated with lentiviral vectors that contain an anti-tat/rev short hairpin RNA (shRNA), an anti-CCR5 ribozyme and a nucleolar-localizing trans-activation response element (TAR) RNA decoy. TAR is a region of secondary RNA structure that is located within the HIV long terminal repeat and is present in all HIV mRNAs. The region prevents the efficient use of the mRNA unless it is bound by the HIV transactivator (Tat) protein. The engineered stem cells then form part of a bone marrow transplant back into the (now) irradiated individual. As a result T cells are produced that are resistant to HIV-1 infection. As with antiHIV chemotherapy, the triple combination approach is used to prevent the build up of resistant viruses.

\section{Implications and future research directions}

Until recently, with the exception of HIV, research into the interactions between RNA viruses and the nucleolus has in general been descriptive and has focused on the identification of viral proteins that localize to the nucleolus and the delineation of NoLSs. The functional implications of these interactions are now being realized, and great progress is being made in 
Reverse genetics

Genetic analysis that proceeds from genotype to phenotype through gene-manipulation techniques. This method allows the production of viruses that have genes derived from cloned cDNA. elucidating why RNA viruses target the nucleolus and the role of the nucleolar localization of viral proteins in virus replication. Through the use of reverse genetics and full-length clone technologies, this research could lead to the development of new recombinant vaccines based on viral proteins that are deficient in nucleolar trafficking and are therefore attenuated in replication. The study of viral interactions with the nucleolus also presents cell biologists with an opportunity to gain new insights into nucleolar processes and functions. For example, as previously discussed, UBF is inactivated in poliovirus-infected cells and, as might be predicted, RNA polymerase I transcription is downregulated ${ }^{53}$. However, in adenovirus-infected cells most nucleolar $\mathrm{UBF}$ is sequestered in replication centres formed by this DNA virus; but in this case, RNA polymerase I transcription is unaffected ${ }^{111}$, therefore casting doubt on the assumption that UBF is required for RNA synthesis.

Both the cell and viral biology of the nucleolus can, and hopefully will, be exploited for the design of novel therapeutic strategies against viral infection. This is best illustrated in forthcoming clinical trials for therapies to combat HIV-1 (REF. 16). So, although the nucleolus was one of the first subcellular structures to be discovered, nucleolar research is still providing new and exciting insights into both viral and cellular biology.
1. Ahlquist, P. Parallels among positive-strand RNA viruses, reverse-transcribing viruses and doublestranded RNA viruses. Nature Rev. Microbiol. 4 371-382 (2006).

2. Lam, Y. W., Trinkle-Mulcahy, L. \& Lamond, A. I. The nucleolus. J. Cell Sci. 118, 1335-1337 (2005).

3. Hernandez-Verdun, D. Nucleolus: from structure to dynamics. Histochem. Cell Biol. 125, 127-137 (2006). Hernandez-Verdun, D., Roussel, P. \& Hernandez-Verdun, D., Roussel, P. \&
Gebrane-Younes, J. Emerging concepts of nucleolar assembly. J. Cell Sci. 115, 2265-2270 (2002).

5. Shaw, P. J. \& Brown, J. W. Plant nuclear bodies. Curr. Opin. Plant Biol. 7, 614-620 (2004).

6. Huang, S., Deerinck, T. J., Ellisman, M. H. \& Spector, D. L. The perinucleolar compartment and transcription. J. Cell Biol. 143, 35-47 (1998).

7. Pederson, T. \& Politz, J. C. The nucleolus and the four ribonucleoproteins of translation. J. Cell Biol. 148 1091-1095 (2000).

8. Klibanov, S. A., O'Hagan, H. M. \& Ljungman, M. Accumulation of soluble and nucleolar-associated p53 proteins following cellular stress. J. Cell Sci. 114 1867-1873 (2001).

9. Daniely, Y., Dimitrova, D. D. \& Borowiec, J. A. Stressdependent nucleolin mobilization mediated by p53nucleolin complex formation. Mol. Cell. Biol. 22 , 6014-6022 (2002).

10. Rubbi, C. P. \& Milner, J. Disruption of the nucleolus mediates stabilization of p53 in response to DNA damage and other stresses. EMBO J. 22, 6068-6077 (2003).

This is one of the key papers that showed the fundamental role of the nucleolus in responding to cellular stress.

11. Yuan, X. et al. Genetic inactivation of the transcription factor TIF-IA leads to nucleolar disruption, cell cycle arrest, and p53-mediated apoptosis. Mol. Cell 19. 77-87 (2005)

12. Leung, A. K. \& Lamond, A. I. The dynamics of the nucleolus. Crit. Rev. Eukaryot. Gene Expr. 13, 39-54 (2003).

13 Pendle, A. F. et al. Proteomic analysis of the Arabidopsis nucleolus suggests novel nucleolar functions. Mol. Biol. Cell 16, 260-269 (2005)

14. Andersen, J. S. et al. Nucleolar proteome dynamics. Nature 433, 77-83 (2005).

15. Andersen, J. S. et al. Directed proteomic analysis of the human nucleolus. Curr. Biol. 12, 1-11 (2002). This paper was the first thorough description of the nucleolar proteome and has led to rapid advancements in our understanding of the dynamic nucleolus.

16. Matthews, D. A. \& Olson, M. O. What's new in the nucleolus? EMBO Rep. 7, 1-4 (2006)

17. Sirri, V., Roussel, P. \& Hernandez-Verdun, D. The AgNOR proteins: qualitative and quantitative change during the cell cycle. Micron. 31, 121-126 (2000).

18. Cokol, M., Nair, R. \& Rost, B. Finding nuclear localization signals. EMBO Rep. 1, 411-415 (2000).

19. Macara, I. G. Transport into and out of the nucleus. Microbiol. Mol. Biol. Rev. 65, 570-594 (2001). A comprehensive review on how proteins traffic into and out of the nucleus and the different pathways and steps involved.

20. Garcia-Bustos, J. Heitman, J \& Hall, M. N. Nuclear protein localization. Biochem. Biochim. Biophys. Acto 1071, 83-101 (1991).
21. Ossareh-Nazari, B., Gwizdek, C. \& Dargemont, C. Protein export from the nucleus. Traffic 2, 684-689 (2001).

22. Carmo-Fonseca, M., Mendes-Soares, L. \& Campos, I. To be or not to be in the nucleolus. Nature Cell Biol. 2, E107-E112 (2000).

A thought-provoking review on current perceptions of the role of the nucleolus in cell biology, such as its regulation of cellular growth.

23. Kubota, S., Copeland, T. D. \& Pomerantz, R. J. Nuclear and nucleolar targeting of human ribosomal protein S25: common features shared with HIV-1 regulatory proteins. Oncogene 18, 1503-1514 (1999).

24. Song, Z. \& Wu, M. Identification of a novel nucleolar localization signal and a degradation signal in SurvivindeltaEx3: a potential link between nucleolus and protein degradation. Oncogene 24, 2723-2734 (2005).

25. Sheng, Z., Lewis, J. A. \& Chirico, W. J. Nuclear and nucleolar localization of 18-kDa fibroblast growth factor- 2 is controlled by C-terminal signals. J. Biol. Chem. 279, 40153-40160 (2004).

26. Russo, G., Ricciardelli, G. \& Pietropaolo, C. Different domains cooperate to target the human ribosomal L7a protein to the nucleus and to the nucleoli. J. Biol. Chem. 272, 5229-5235 (1997).

27. Michael, W. M \& Dreyfuss, G. Distinct domains in ribosomal protein L5 mediate $5 \mathrm{~S}$ rRNA binding and nucleolar localization. J. Biol. Chem. 271, 11571-11574 (1996)

28. Tsai, R. Y. L. \& McKay, R. D. G. A multistep, GTP-driven mechanism controlling the dynamic cycling of nucleostemin. J. Cell Biol. 168, 179-184 (2005).

29. Cros, J. F., Garcia-Sastre, A. \& Palese, P. An unconventional NLS is critical for the nuclear import of the influenza A virus nucleoprotein and ribonucleoprotein. Traffic 6, 205-213 (2005).

30. Tsukahara, F. \& Maru, Y. Identification of novel nuclear export and nuclear localization-related signals in human heat shock cognate protein 70. J. Biol. Chem 279, 8867-8872 (2004).

31 Ryabov, E. V., Kim, S. H. \& Taliansky, M. Identification of a nuclear localization signal and nuclear export signal of the umbraviral long-distance RNA movement protein. J. Gen. Virol. 85, 1329-1333 (2004).

32. Ladd, A. N. \& Cooper, T. A. Multiple domains control the subcellular localization and activity of ETR-3, a regulator of nuclear and cytoplasmic RNA processing events. J. Cell Sci. 117, 3519-3529 (2004).

33. Wilhelmsen, K. C., Leibowitz, J. L., Bond, C. W. \& Robb, J. A. The replication of murine coronaviruses in enucleated cells. Virology 110, 225-230 (1981).

34. Evans, M. R. \& Simpson, R. W. The coronavirus avian infectious bronchitis virus requires the cell nucleus and host transcription factors. Virology 105, 582-591 (1980).

35. Hiscox, J. A. et al. The coronavirus infectious bronchitis virus nucleoprotein localizes to the nucleolus. J. Virol. 75, 506-512 (2001).

36. Wurm, T., Chen, H., Britton, P., Brooks, G. $\&$ Hiscox, J. A Localisation to the nucleolus is a common feature of coronavirus nucleoproteins and the protein may disrupt host cell division. J. Virol. 75, 9345-9356 (2001)

37. Dove, B. K. et al. Changes in nucleolar architecture and protein profile during coronavirus infection. Cell. Microbiol. 8, 1147-1157 (2006).
38. You, J.-H. et al. Sub-cellular localisation of the severe acute respiratory syndrome coronavirus nucleocapsid protein. J. Gen. Virol. 86, 3303-3310 (2005).

39. Timani, K. A. et al. Nuclear/nucleolar localization properties of C-terminal nucleocapsid protein of SARS coronavirus. Virus Res. 114, 23-34 (2005).

40. Tijms, M. A., van der Meer, Y. \& Snijder, E. J. Nuclear localization of non-structural protein 1 and nucleocapsid protein of equine arteritis virus. J. Gen Virol. 83, 795-800 (2002).

41. Rowland, R. R., Kerwin, R., Kuckleburg, C., Sperlich, A $\&$ Benfield, D. A. The localisation of porcine reproductive and respiratory syndrome virus nucleocapsid protein to the nucleolus of infected cells and identification of a potential nucleolar localization signal sequence. Virus Res. 64, 1-12 (1999).

42. Michel, M. R. et al. Karyophilic properties of Semliki Forest virus nucleocapsid protein. J. Virol. 64 , 5123-5131 (1990)

43. Rikkonen, M., Peranen, J. \& Kaariainen, L. Nuclear and nucleolar targeting signals of Semliki Forest virus nonstructural protein nsP2. Virology 189, 462-473 (1992).

44. Wang, S. H. et al. Intracellular localization and determination of a nuclear localization signal of the core protein of dengue virus. J. Gen. Virol. 83, 3093-3102 (2002).

45. Taliansky, M. E. \& Robinson, D. J. Molecular biology of umbraviruses: phantom warriors. J. Gen. Virol. 84, 1951-1960 (2003).

46. Haupt, S. et al. Nucleolar localization of potato leafroll virus capsid proteins. J. Gen. Virol. 86, 2891-2896 (2005).

47. Peeples, M. E., Wang, C., Gupta, K. C. \& Coleman, N. Nuclear entry and nucleolar localization of the Newcastle disease virus (NDV) matrix protein occur early in infection and do not require other NDV proteins. J. Virol. 66, 3263-3269 (1992)

48. Dimmock, N. J. New virus-specific antigens in cells infected with influenza virus. Virology 39, 224-234 (1969).

49. Compans, R. W. \& Dimmock, N. J. An electron microscopic study of single-cycle infection of chick embryo fibroblasts by influenza virus. Virology 39 , 499-515 (1969)

50. Hiscox, J. A. The nucleolus - a gateway to viral infection? Arch Virol 147, 1077-1089 (2002).

51. Hancock, R. A role for macromolecular crowding effects in the assembly and function of compartments in the nucleus. J. Struct. Biol. 146, 281-290 (2004).

52. Waggoner, S. \& Sarnow, P. Viral ribonucleoprotein complex formation and nucleolar-cytoplasmic relocalization of nucleolin in poliovirus-infected cells. J. Virol. 72, 6699-6709 (1998)

53. Banerjee, R., Weidman, M. K., Navarro, S., Comai, L. \& Dasgupta, A. Modifications of both selectivity factor and upstream binding factor contribute to poliovirusmediated inhibition of RNA polymerase I transcription. J. Gen. Virol. 86, 2315-2322 (2005)

54. Dove, B. K., Brooks, G., Bicknell, K. A., Wurm, T. \& Hiscox, J. A. Cell cycle perturbations induced by infection with the coronavirus infectious bronchitis virus and their effect on virus replication. J. Virol. 80 4147-4156 (2006)

55. Chen, H., Wurm, T., Britton, P., Brooks, G. \& Hiscox, J. A. Interaction of the coronavirus nucleoprotein with nucleolar antigens and the host cell. J. Virol. 76, 5233-5250 (2002) 
56. Yoo, D., Wootton, S. K., Li, G., Song, C. $\delta$ Rowland, R. R. Colocalization and interaction of the porcine arterivirus nucleocapsid protein with the small nucleolar RNA-associated protein fibrillarin. J. Virol. 77 12173-12183 (2003).

57. Miyazaki, Y., Nosaka, T. \& Hatanaka, M. The posttranscriptional regulator Rev of HIV: implications for its interaction with the nucleolar protein B23. Biochimie 78, 1081-1086 (1996).

58. Cannavo, G. et al. Abnormal intracellular kinetics of cellcycle-dependent proteins in lymphocytes from patients infected with human immunodeficiency virus: a novel biologic link between immune activation, accelerated T-cell turnover, and high levels of apoptosis. Blood 97 1756-1764 (2001)

59. Galati, D. et al. Specific changes in the posttranslational regulation of nucleolin in lymphocytes from patients infected with human immunodeficiency virus. J. Infect. Dis. 188, 1483-1491 (2003).

60. Paiardini, M. et al. Exogenous interleukin-2 administration corrects the cell cycle perturbation of lymphocytes from human immunodeficiency virusinfected individuals. J. Virol. 75, 10843-10855 (2001)

61. Rowland, R. R. \& Yoo, D. Nucleolar-cytoplasmic shuttling of PRRSV nucleocapsid protein: a simple case of molecular mimicry or the complex regulation by nuclear import, nucleolar localization and nuclear export signal sequences. Virus Res. 95, 23-33 (2003).

62. Kalderon, D., Roberts, B. L., Richardson, W. D. \& Smith, A. E. A short amino acid sequence able to specify nuclear location. Cell 39, 499-509 (1984).

63. Rowland, R. R. et al. Peptide domains involved in the localization of the porcine reproductive and respiratory syndrome virus nucleocapsid protein to the nucleolus. Virology 316, 135-145 (2003).

64. Lee, C. H., Chang, S. C., Chen, C. J. \& Chang, M. F. The nucleolin binding activity of hepatitis delta antigen is associated with nucleolus targeting. J. Biol. Chem. 273 7650-7656 (1998).

65. Marra, M. A. et al. The genome sequence of the SARS associated coronavirus. Science 300, 1399-1404 (2003).

66. Reed, M. et al. Delineation and modelling of a novel nucleolar retention signal in the coronavirus nucleocapsid protein. Traffic 7, 833-849 (2006).

67. Rowland, R. R. et al. Intracellular localization of the severe acute respiratory syndrome coronavirus nucleocapsid protein: absence of nucleolar accumulation during infection and after expression as a recombinant protein in vero cells. J. Virol. 79, 11507-11512 (2005)

68. Peranen, J., Rikkonen, M., Liljestrom, P. \& Kaariainen, L. Nuclear localization of Semliki Forest virus-specific nonstructural protein nsP2. J. Virol. 64, 1888-1896 (1990).

69. Fazakerley, J. K., Boyd, A., Mikkola, M. L. \& Kaariainen, L. A single amino acid change in the nuclear localization sequence of the nsP2 protein affects the neurovirulence of Semliki forest virus. J. Virol. 76, 392-396 (2002).

70. Lee, C. et al. Mutations within the nuclear localization signal of the porcine reproductive and respiratory syndrome virus nucleocapsid protein attenuate virus replication. Virology 346, 238-250 (2006) This paper was the first to convincingly show that nuclear targeting of viral capsid proteins has an important role in the life cycle of a positive-strand RNA virus. It was particularly informative as the work was conducted in vivo, as well as in vitro.

71. Mori, Y. et al. Nuclear localization of Japanese encephalitis virus core protein enhances viral replication J Virol 79, 3448-3458 (2005).

72. Thiry, M. \& Lafontaine, D. L. Birth of a nucleolus: the evolution of nucleolar compartments. Trends Cell Biol. 15, 194-199 (2005).

A thought-provoking and perhaps controversial review of the structure and evolution of the nucleolus.

73. Tsuda, Y. et al. Nucleolar protein B23 interacts with Japanese encephalitis virus core protein and participates in viral replication. Microbiol. Immunol. 50, 225-234 (2006)

74. Uchil, P. D., Kumar, A. V. \& Satchidanandam, V. Nuclear localization of flavivirus RNA synthesis in infected cells. J. Virol. 80, 5451-5464 (2006).

75. Cole, C. N. \& Scarcelli, J. J. Transport of messenger RNA from the nucleus to the cytoplasm. Curr. Opin. Cell Biol. 18, 299-306 (2006).

76. Goodwin, D. J., Hall, K. T., Stevenson, A. J. Markham, A. F. \& Whitehouse, A. The open reading frame 57 gene product of herpesvirus saimiri shuttles between the nucleus and cytoplasm and is involved in viral RNA nuclear export. J. Virol. 73, 10519-10524 (1999).
77. Goodwin, D. J. \& Whitehouse, A. A $\gamma-2$ herpesvirus nucleocytoplasmic shuttle protein interacts with importin $\alpha 1$ and $\alpha 5$. J. Biol. Chem. 276 19905-19912 (2001)

78. Williams, B. J. et al. The prototype $\gamma-2$ herpesvirus nucleocytoplasmic shuttling protein, ORF 57 transports viral RNA through the cellular mRNA export pathway. Biochem. J. 387, 295-308 (2005)

79. Uhrig, J. F., Canto, T., Marshall, D. \& MacFarlane, S. A. Relocalization of nuclear ALY proteins to the cytoplasm by the tomato bushy stunt virus $\mathrm{P} 19$ pathogenicity protein. Plant Physiol. 135, 2411-2423 (2004).

80. Canto, T., Uhrig, J. F., Swanson, M., Wright, K. M. \& MacFarlane, S. A. Translocation of tomato bushy stunt virus P19 protein into the nucleus by ALY proteins compromises its silencing suppressor activity. J. Virol. 80, 9064-9072 (2006).

81. Harders, J., Lukacs, N., Robert-Nicoud, M., Jovin, T. M. \& Riesner, D. Imaging of viroids in nuclei from tomato leaf tissue by in situ hybridization and confocal laser scanning microscopy. EMBO J. 8, 3941-3949 (1989).

82. Ryabov, E. V., Oparka, K. J., Santa Cruz, S., Robinson, D. J. \& Taliansky, M. E. Intracellular location of two groundnut rosette umbravirus proteins delivered by PVX and TMV vectors. Virology 242, 303-313 (1998).

83. Tsai, C. W. et al. Complete genome sequence and in planta subcellular localization of maize fine streak virus proteins. J. Virol. 79, 5304-5314 (2005).

84. Kim, S. H., Ryabov, E. V., Brown, J. W. \& Taliansky, M. Involvement of the nucleolus in plant virus systemic infection. Biochem. Soc. Trans. 32, 557-560 (2004).

85. Pyper, J. M., Clements, J. E. \& Zink, M. C. The nucleolus is the site of Borna disease virus RNA transcription and replication. J. Virol. 72, 7697-7702 (1998).

86. Cros, J. F. \& Palese, P. Trafficking of viral genomic RNA into and out of the nucleus: influenza, Thogoto and Borna disease viruses. Virus Res. 95, 3-12 (2003).

87. Li, Y. J., Macnaughton, T., Gao, L. \& Lai, M. M. RNA-templated replication of hepatitis delta virus: genomic and antigenomic RNAs associate with different nuclear bodies. J. Virol. 80, 6478-6486 (2006).

88. Qi, Y. \& Ding, B. Differential subnuclear localization of RNA strands of opposite polarity derived from an autonomously replicating viroid. Plant Cell 15 2566-2577 (2003)

89. Izumi, R. E., Valdez, B., Banerjee, R., Srivastava, M. \& Dasgupta, A. Nucleolin stimulates viral internal ribosome entry site-mediated translation. Virus Res. 76, 17-29 (2001).

90. Hellen, C. U. T. \& Sarnow, P. Internal ribosome entry sites in eukaryotic mRNA molecules. Genes Dev. 15 1593-1612 (2001)

91. Hirano, M. et al. Direct interaction between nucleolin and hepatitis C virus NS5B. J. Biol. Chem. 278 5109-5115 (2003)

92. Amineva, S. P., Aminev, A. G., Palmenberg, A. C. \& Gern, J. E. Rhinovirus $3 C$ protease precursors $3 C D$ and $3 C D^{\prime}$ localize to the nuclei of infected cells. J. Gen. Virol. 85, 2969-2979 (2004).

93. Aminev, A. G., Amineva, S. P. \& Palmenberg, A. C. Encephalomyocarditis viral protein $2 A$ localizes to nucleoli and inhibits cap-dependent mRNA translation. Virus Res. 95, 45-57 (2003).

94. Aminev, A. G., Amineva, S. P. \& Palmenberg, A. C Encephalomyocarditis virus (EMCV) proteins $2 \mathrm{~A}$ and 3BCD localize to nuclei and inhibit cellular mRNA transcription but not rRNA transcription. Virus Res. 95 59-73 (2003)

95. Pisitkun, P. et al. Autoreactive B cell responses to RNArelated antigens due to TLR7 gene duplication. Science 312, 1669-1672 (2006)

96. Seko, Y., Cole, S., Kasprzak, W., Shapiro, B. A. \& Ragheb, J. A. The role of cytokine mRNA stability in the pathogenesis of autoimmune disease. Autoimmun. Rev. 5, 299-305 (2006)

97. Perkins, A., Cochrane, A. W., Ruben, S. M. \& Rosen, C. A Structural and functional characterization of the human immunodeficiency virus rev protein. J. Acquir. Immune Defic. Syndr. 2, 256-263 (1989).

98. Ruben, S. et al. Structural and functional characterization of human immunodeficiency virus Ta protein. J. Virol. 63, 1-8 (1989).

99 Sanchez-Velar, N., Udofia, E. B., Yu, Z. \& Zapp, M. L. hRIP, a cellular cofactor for Rev function, promotes release of HIV RNAs from the perinuclear region. Genes Dev. 18, 23-34 (2004)

100. Venkatesh, L. K., Mohammed, S. \& Chinnadurai, G. Functional domains of the HIV-1 rev gene required for trans-regulation and subcellular localization. Virology 176, 39-47 (1990).
101. Cochrane, A. W., Perkins, A. \& Rosen, C. A. Identification of sequences important in the nucleolar localization of human immunodeficiency virus Rev relevance of nucleolar localization to function. J. Virol. 64, 881-885 (1990).

102. Dundr, M. et al. The roles of nucleolar structure and function in the subcellular location of the HIV-1 Rev protein. J. Cell Sci. 108, 2811-2823 (1995).

103. Daelemans, D. et al. In vivo HIV-1 Rev multimerization in the nucleolus and cytoplasm identified by fluorescence resonance energy transfer. J. Biol. Chem. 279, 50167-50175 (2004).

104. Michienzi, A., De Angelis, F. G., Bozzoni, I. \& Rossi, J. J. A nucleolar localizing Rev binding element inhibits HIV replication. AIDS Res. Ther. 3, 13 (2006).

Together with other papers from this group, this is groundbreaking research into how disrupting viral-nucleolar interactions can lead to novel and hopefully successful anti-HIV therapeutic strategies.

105 Siomi, H. Shida, H., Maki, M. \& Hatanaka, M. Effects of a highly basic region of human immunodeficiency virus Tat protein on nucleolar localization. J. Virol. 64 1803-1807 (1990)

106. Michienzi, A., Li, S., Zaia, J. A. \& Rossi, J. J. A nucleolar TAR decoy inhibitor of HIV-1 replication. Proc. Natl Acad. Sci. USA 99, 14047-14052 (2002).

107. Unwalla, H. J. et al. Negative feedback inhibition of HIV-1 by TAT-inducible expression of siRNA. Nature Biotechnol. 22, 1573-1578 (2004).

108. Michienzi, A., Cagnon, L., Bahner, I. \& Rossi, J. J. Ribozyme-mediated inhibition of HIV 1 suggests nucleolar trafficking of HIV-1 RNA. Proc. Natl Acad. Sci. USA 97, 8955-8960 (2000).

109. Li, M. J. et al. Long-term inhibition of HIV-1 infection in primary hematopoietic cells by lentiviral vector delivery of a triple combination of anti-HIV shRNA, anti-CCR5 ribozyme, and a nucleolar-localizing TAR decoy. Mol. Ther. 12, 900-909 (2005)

110 Oin, X. F. An, D. S., Chen, I. S. \& Baltimore, D. Inhibiting HIV-1 infection in human T cells by lentiviralmediated delivery of small interfering RNA against CCR5. Proc. Natl Acad. Sci. USA 100, 183-188 (2003).

111 Lawrence, F. J., McStay, B. \& Matthews, D. A Nucleolar protein upstream binding factor is sequestered into adenovirus DNA replication centres during infection without affecting RNA polymerase I location or ablating rRNA synthesis. J. Cell Sci. 119 2621-2631 (2006)

This work illustrates how a study of viral interactions with the nucleolus can lead to shifts in our understanding of cell biology.

112. Whelan, S. P., Barr, J. N. \& Wertz, G. W. Transcription and replication of nonsegmented negative-strand RNA viruses. Curr. Top. Microbiol. Immunol. 283, 61-119 (2004).

113. Phair, R. D. \& Misteli, T. High mobility of proteins in the mammalian cell nucleus. Nature 404, 604-609 (2000).

114. Chen, D. \& Huang, S. Nucleolar components involved in ribosome biogenesis cycle between the nucleolus and nucleoplasm in interphase cells. J. Cell Biol. 153 169-176 (2001)

115. Dundr, M., Misteli, T. \& Olson, M. O. J. The dynamics of postmitotic reassembly of the nucleolus. J. Cell Biol. 150, 433-446 (2000).

\section{Acknowledgements}

The author would like to thank D. Matthews (University of Bristol), R. Fearns (University of Dundee) and B. Dove (Health Protection Agency) for critically reviewing the manuscript and J. Rossi (Beckman Research Institute) for insights on targeting the nucleolus to disrupt virus infection. Electron microscopy images of the nucleolus in Figure 1 were kindly provided by D. Hernandez-Verdun (Institut Jacques Monod) and the image in Box 1 by T. Misteli (NIH)

Competing interests statement

The author declares no competing financial interests.

DATABASES

The following terms in this article are linked online to: Entrez Genome: http://www.ncbi.nlm.nih.gov/entrez/query. fcgi?db=genome

Borna disease virus | HIV-1 | Japanese encephalitis virus | SARS-CoV | tomato bushy stunt virus | West Nile virus Access to this links box is available online. 